\section{Urinary hemosiderin: role in evaluation of chronic venous insufficiency}

\author{
Ashish Lal Shrestha, ${ }^{1}$ Indrani Sen, ${ }^{1}$ \\ Edwin Stephen, ${ }^{1}$ Prabhu Premkumar, ${ }^{1}$ \\ Sunil Agarwal, ${ }^{1}$ Sukesh Chandran ${ }^{2}$ \\ 'Department of Vascular Surgery and \\ ${ }^{2}$ Department of Transfusion medicine, the \\ Christian Medical College, Vellore, India
}

\section{Abstract}

Chronic venous insufficiency (CVI) leads to skin changes with dermal hemosiderin deposition. We studied the presence of hemosiderin in the urine to assess if this could be used as a biochemical marker for CVI. Hereby we present a case control study conducted in a tertiary care centre in South India. There were 100 cases with evidence of advanced CVI (the Clinical-Etiology-Anatomy-Pathophysiology classification: C5, C6) confirmed by duplex scanning. Controls were 50 patients with leg ulcers due to other etiologies. All patients were subjected to urinary hemosiderin testing. In all 100 patients with CVI (C5 and C6 disease) axial venous reflux was confirmed by duplex ultrasound. Superficial venous reflux was noted in $71 \%$ of patients and deep venous reflux in 54.\%. Primary venous insufficiency was the etiology in $81 \%$ of patients. Only $4 / 100$ patients had detectable amounts of hemosiderin in the urine. Urine hemosiderin testing to determine presence or absence of CVI yielded the following values: positive predictive value- $80 \%$; negative predictive value- $33 \%$; sensitivity-4\% and specificity-98\%. The test could not be recommended as a marker of CVI. In Indian patients urinary hemosiderin is not a useful screening test in CVI.

\section{Introduction}

Varicose veins is a common problem in India, the overall prevalence is about 6 to $25 \%$ in the adult male population. ${ }^{1}$ Presentation with chronic venous insufficiency (skin changes, ulcers) is common. However the incidence and management outcomes are poorly reported. Management is complicated by a low awareness about the nature of the disease, limited availability of health care resources and the long distances patients need to travel to access health care. A simple test to detect chronic venous disease and differentiate it from other causes of lower extremity ulcers would be extremely useful in management. It would help primary physicians in the community to appropriately select patients for referral to centers for intervention for varicose veins.

The presence of hemosiderin in the urine is reported to be a sensitive, cost effective, non invasive and repeatable test that enables detection and progression of microcirculatory overload in patients with chronic venous insufficiency (CVI). It is also reported to differentiate venous ulcers from ischemic ulcers. Hemosiderinuria score enables classification of clinical severity and is useful in follow up of intervention. ${ }^{2,3}$ We studied the presence of hemosiderin in the urine to assess if this could be used as a biochemical marker.

\section{Materials and Methods}

This study was conducted in Christian Medical College and Hospital, Vellore, an 1800 bed tertiary care centre and teaching institute in South India. It was a prospective case control study. At the start of the study, a sample size of 288 (144 cases and 144 controls) was determined using the results reported in two previous studies. In due course, since most of the tests were negative, the sample size was recalculated and 100 cases and 50 controls were studied. Informed consent was obtained from all cases and controls in a language that they understood. The duration of the study was from September 2008 to August 2010. Study cases were patients with leg ulcers with skin changes suggestive of CVI and venous insufficiency confirmed by duplex ultrasound (C5, C6). Controls were patients with leg ulcers of other etiology with no evidence of venous insufficiency on duplex. Patients with previous venous surgery, intravascular hemolytic state and lymphedema were excluded from the study.

The demographic profile of the patients was studied and the clinical features recorded using the Clinical-Etiology-Anatomy-Pathophysiology classification (CEAP) classification. Urinary hemosiderin was tested in single $20 \mathrm{~mL}$ early morning urine, which was centrifuged at $1200 \mathrm{~g}$ for $10-15 \mathrm{~min}$. The remnant was mixed with Perl's reagent containing potassium ferrocyanide and hydrochloric acid and centrifuged for $5 \mathrm{~min}$. Then the sample was studied under a light microscope to detect hemosiderin crystals. Haemosiderin, if present, appears in the form of isolated or grouped blue-staining granules, usually from 1 to $3 \mu \mathrm{m}$ in size, they may be both intracellular and extracellular.

The laboratory personnel were not given information about the study.
Correspondence: Sunil Agarwal, Department of Vascular Surgery, Christian Medical College, Vellore 632 004, India. Tel. +91.416.2282085.

E-mail:vascular@cmcvellore.ac.in

Key words: urinary haemosiderin, chronic venous insufficiency.

Received for publication: 4 April 2012.

Revision received: 3 August 2012.

Accepted for publication: 6 August 2012

This work is licensed under a Creative Commons Attribution 3.0 License (by-nc 3.0).

(C) Copyright A.L. Shrestha et al., 2012

Licensee PAGEPress, Italy

Veins and Lymphatics 2012; 1:e5

doi:10.4081/vl.2012.e5

\section{Statistical analysis}

Data were collected and analyzed with Statistical Package for the Social Sciences (SPSS) for windows version 15.0 (SPSS Inc., Chicago, IL, USA). Descriptive statistics were calculated using the SPSS software. Comparisons between individual groups of patients were made using Chi square test where appropriate.

\section{Results}

One hundred patients with CVI were included in this study. Fifty five percent were in the age group 21-50 and 44\% were 51 and above. There were 86 male and 14 female patients. The mean duration of symptoms at presentation was 6 years (range 1 month to 20 years). The average age at presentation was 57 years (range 20-79). The control group had a similar age and sex distribution, there were 45 male and 5 female patients, and the average age was 52 years. The haemosiderin positive patients did not belong to the same ethnic group. Out of 100 cases with CVI 79\% had C6 disease and 21\% had C5 disease. On duplex ultrasound, $71 \%$ had superficial reflux, $64 \%$ had deep reflux, $8 \%$ had a combination of both. The Saphenofemoral junction (SFJ) was incompetent in $71 \%$ and saphenopopliteal junction (SPJ) in 16\%. Isolated SFJ incompetence was seen in $10 \%$. There were no patients with isolated SPJ reflux. The distribution of the patients according to the CEAP classification and distribution of venous reflux is given in Table 1. Duplex ultrasound demonstrated evidence of prior deep venous thrombosis (DVT)/partial obstruction in $19 \%$ of patients. Primary venous insufficiency was diagnosed in $81 \%$ of patients and postthrombotic syn- 
Table 1. Distribution of patients in severe chronic venous insufficiency (Clinical-Etiology-Anatomy-Pathophysiology classification: C5, C6).

$\begin{array}{lccccccccc} & \text { C5 } & \text { C6 } & \text { Primary } & \text { Secondary } & \begin{array}{c}\text { No. of cases } \\ \text { Superficial }\end{array} & \text { Deep } & \text { Perforator } & \text { Reflux } & \text { Obstruction } \\ \text { Clinical } & 21 & 79 & & & & & & & \\ \text { Etiological } & & & 81 & 19 & & & & & \\ \text { Anatomy } & & & & & 71 & 64 & 79 & 54 & 19 \\ \text { Pathology } & & & & & & & 9\end{array}$

drome in 19\%. One hundred cases and 50 controls underwent urinary testing for haemosiderin; the results are shown in Table 2. Based on the above results and $2 \times 2$ table, sensitivity of $4 \%$, specificity of $2 \%$, a positive predictive value of $80 \%$ and a negative predictive value of $66 \%$ were obtained. After cross tabulation and Pearson Chi Square testing, a P value of 0.414 was obtained which was statistically insignificant.

\section{Discussion}

Chronic venous insufficiency is a term used to describe any chronic disorder of the veins of the limbs, this represents those patients who have developed irreversible skin changes as the result of sustained ambulatory venous hypertension. Although the exact prevalence of CVI in India is not known, it is common and majority of patients present late with severe disease. This is evident from a high incidence of untreated superficial reflux (71\%) in these patients. The duration of symptoms did not correlate with severity of disease as few patients with ulcers reported onset as less as a month. The average age at presentation with ulceration is at a younger age than reported in the west and this makes it a significant economic burden. The increased incidence in men might be due to reluctance on the part of women to seek medical attention. A large percentage had deep venous reflux on duplex ultrasound and in $12 \%$ this was associated with untreated superficial disease. This indicates the need for early intervention in patients with deep reflux. A significant percentage presented with sequelae of undiagnosed DVT: awareness about this also needs to be increased.

In chronic venous insufficiency the characteristic skin changes are attributed to dermal deposition of hemosiderin. Chronic venous stasis and impaired venous drainage is followed by microcirculatory overload leading to erythrocyte diapedesis, red blood cell extravasation into the surrounding tissue and subsequent hemolysis and dermal ferritin deposition. This ultimately gets converted to dermal

Table 2. Results of urinary hemosiderin test.

\begin{tabular}{|c|c|c|}
\hline \multirow[b]{2}{*}{ Test CVI } & \multicolumn{2}{|c|}{ No. of cases } \\
\hline & Negative & Positive \\
\hline Present & 96 & 4 \\
\hline Absent & 49 & 1 \\
\hline
\end{tabular}

$\mathrm{CVI}$, chronic venous insufficiency.

hemosiderin thereby giving characteristic skin changes. This dermal and tissue hemosiderin is thought to be taken into the circulation via the lymphatics and gets deposited in the tubular epithelium and then excreted in urine.

Use of hemosiderinuria in CVI is a controversial issue with various unclear aspects mainly in condition of iron overload disease. In their paper, Zamboni et al. show a correspondence between the test and the strictness of the disease. This point of view was confirmed also by other authors ${ }^{3}$ though without a statistically meaningful correlation between the tissue accumulation of hemosiderin and the clinical trials. The test can be positive even in other diseases such as lymphedema and connective tissue disease. ${ }^{3}$ Hemosiderinuria represents a marker for intravascular hemolysis, polymorphonuclear leukocytes are able to englobe hemosiderin granules and show them in the urine. Hemosiderin storage in the ulcer margin tissue also occurs in CVI. ${ }^{4}$

However there may be population differences in the renal elimination pattern and this may explain why our patients do not have hemosiderinuria.

Familial venous disease has an underlying genetic basis with a postulated autosomal dominant pattern with variable penetrance. Mutations in the haemochromotosis gene (HFE) C $282 Y$ mutation significantly increase rates of CVI. Factor XIII gene variants are also associated with venous disease. These mutations and gene variants are thought to be associated with development, healing and recurrence of venous ulcers. The HFE mutations are thought to result in a less efficient transport of iron by macrophages; increase excretion and are thought to increase leg ulceration. The mutations though not studied in CVI, are thought to be rare in the Indian population. ${ }^{5-14}$ This may explain the absence of accumulation and excretion of haemosiderin-alternative disease causation is thus postulated as a mechanism of disease.

\section{Conclusions}

Chronic venous insufficiency is a common problem in the Indian population and patients frequently present late. The vast majority has untreated superficial reflux and would potentially benefit from superficial venous reflux ablation. A simple screening test is needed to diagnose CVI in the community and aid in selecting patients for referral to higher centers for venous intervention. However, testing for urinary hemosiderin does not reliably differentiate CVI from lower extremity ulceration from other causes in this patient population.

\section{References}

1. Malhotra SL. An epidemiological study of varicose veins in Indian railroad workers from the South and North of India, with special reference to the causation and prevention of varicose veins. Int $\mathrm{J}$ Epid 1972;1:177-83.

2. Zamboni P, Izzo M, Fogato L, et al. Urinary haemosiderin: a novel marker to assess the severity of chronic venous disease. J Vasc Surg 2003;37:132-6.

3. Tan J, Smith A, Abisi S, et al. Tissue and urinary haemosiderin in chronic leg ulcers. Eur J Vasc Endovasc Surg 
2007;34:355-60.

4. Caggiati A, Rosi C, Casini A, et al. Skin iron deposition characterises lipodermatosclerosis and leg ulcer. Eur J Vasc Endovasc Surg 2010;40:777-82.

5. Dhillon BK, Das R, Garewal G, et al. Frequency of primary iron overload and HFE gene mutations (C282Y, H63D and $\mathrm{S} 65 \mathrm{C}$ ) in chronic liver disease patients in North India. World J Gastroenterol 2007;13:2956-9.

6. Jain S, Agarwal S, Tamhankar P, et al. Lack of association of primary iron overload and common HFE gene mutations with liver cirrhosis in adult Indian population. Indian J Gastroenterol 2011;30:161-5.

7. Sindrilaru A, Peters T, Wieschalka S, et al. An unrestrained proinflammatory M1 macrophage population induced by iron impairs wound healing in humans and mice. J Clin Invest 2011;121:985.

8. Zamboni P, Tognazzo S, Izzo M, et al. Hemochromatosis C282Y gene mutation increases the risk of venous leg ulceration. J Vasc Surg 2005;42:309-14.

9. Zamboni P, Izzo M, Tognazzo S, et al. The overlapping of local iron overload and HFE mutation in venous leg ulcer pathogenesis. Free Radic Biol Med 2006;40:1869-73.

10. Simka M, Rybak Z. Hypothetical molecular mechanisms by which local iron overload facilitates the development of venous leg ulcers and multiple sclerosis lesions. Med Hypotheses 2008;71:293-7.

11. Gemmati D, Federici F, Catozzi L, et al. DNA-array of gene variants in venous leg ulcers: detection of prognostic indicators. J Vasc Surg 2009;50:1444-51.

12. Gemmati D, Tognazzo S, Catozzi L, et al. Influence of gene polymorphisms in ulcer healing process after superficial venous surgery. J Vasc Surg 2006;44:554-62.

13. Tognazzo S, Gemmati D, Palazzo A, et al. Prognostic role of factor XIII gene variants in nonhealing venous leg ulcers. J Vasc Surg 2006;44:815-9.

14. Cornu-Thenard A, Boivin P, Baud JM, et al. Importance of the familial factor in varicose disease. Clinical study of 134 families. J Dermatol Surg Oncol 1994;20:31826. 